\title{
GrußWOrt anlässlich der Amtseinführung von Herrn Dr. Rupert Schaab in das Amt des Direktors der Württembergischen Landesbibliothek am 26.11.2019
}

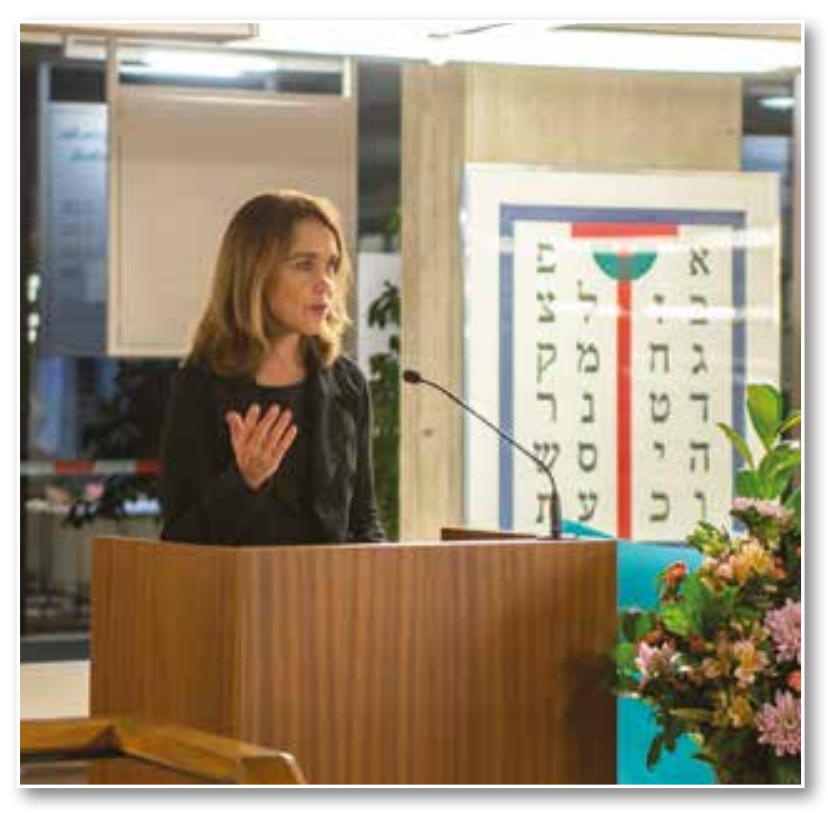

Sehr geehrte Damen und Herren, lieber Herr Schaab, ich freue mich, heute zu Ihrer Amtseinführung hier zu sein. Sie haben bereits im Juni dieses Jahres die Leitung der Württembergischen Landesbibliothek übernommen und daher sind wir uns bereits mehrfach auf Veranstaltungen - auch hier im Haus - begegnet. Dieses Haus, die Württembergische Landesbibliothek, und Ihr neues Team konnten Sie in den letzten Monaten kennenlernen. Sie haben testen können, welche Systeme und Arbeitsmethoden funktionieren und sich bewährt haben, und was man auch verändern oder erneuern kann. Was dazu geführt hat, dass Sie gerade an einem Konzept zur Weiterentwicklung der Landesbibliothek arbeiten, auf das wir alle sehr gespannt sind. Seien Sie versichert, dass wir von Seiten des Ministeriums für Wissenschaft, Forschung und Kunst alles daransetzen werden, Sie bei der Bewältigung der vielfältigen Aufgaben zu unterstützen.

Aber wie Sie in den letzten Monaten schon erfahren konnten, die wichtigste Voraussetzung hierfür ist bereits gegeben: Sie übernehmen ein gut bestelltes Haus mit einem engagierten Mitarbeiterteam. Erlauben Sie mir an dieser Stelle, der stell- vertretenden Direktorin, Frau Martina Lüll, für ihren äußerst verdienstvollen Einsatz in diesen vergangenen Monaten herzlich zu danken. Sie haben die Belange der Bibliothek engagiert vertreten und das Haus sicher durch diese Zeit gelenkt. Sie werden, lieber Herr Schaab, sicherlich vielfältige Unterstützung durch Ihre Stellvertreterin erfahren, durch Ihre übrigen Mitarbeiterinnen und Mitarbeiter, durch Ihre Kolleginnen und Kollegen im Land und durch den Förderverein der Württembergischen Landesbibliothek, die Württembergische Bibliotheksgesellschaft. Fördervereine sind in unserer Zeit äußerst wichtige Einrichtungen und ich freue mich, dass die Württembergische Bibliotheksgesellschaft sich stets mit vollem Einsatz für die Sache der Württembergischen Landesbibliothek eingesetzt hat.

Und die WLB ist das Engagement auf jeden Fall wert! Denn die größte Universalbibliothek des Landes bietet mit ihrer umfangreichen Spannbreite von wertvollen Altbeständen bis hin zur digitalen Bibliothek enorme Möglichkeiten nicht nur im Servicebereich, sondern auch bei der digitalen Vermittlung und der kulturellen Bildung. Mit ihrer mehr als 250 Jahre alten Geschichte ist die WLB zugleich eine bedeutende Kulturinstitution, die mit ihrem Kulturangebot weit über Stuttgart hinaus in die ganze Region hinein wirkt.

Unsere Aufgabe - und von nun an auch die Ihre, lieber Herr Schaab, - ist es, diese Außenwirkung weiter zu verstärken. Ich sehe eine der ganz großen Zukunftsaufgaben darin, unsere wertvollen kulturellen Institutionen der breiteren Gesellschaft weiter zu öffnen. Häuser wie die Württembergische Landesbibliothek müssen von uns - und damit meine ich auch die Politik - in Zukunft weiter gestärkt werden, damit ihre reichhaltigen und spannenden Inhalte noch viel mehr Menschen als bisher erreichen, und damit sie als Orte des Wissens und des lebenslangen Lernens die ganze Breite und Vielfalt unserer Gesellschaft ansprechen und erreichen können. 
Über das Konzept der sogenannten "dritten Orte" wird viel gesprochen, und man muss mittlerweile fast schon aufpassen, dass die Idee dieser "dritten Orte" nicht zum Schlagwort verkommt. Trotzdem halte ich den Grundgedanken, dass Kultureinrichtungen (und somit auch Bibliotheken) viel mehr sein können, und auch viel mehr sein müssen, als reine Lese- und Forschungsorte, für essentiell. Die Landesbibliothek der Zukunft muss ein Ort der Begegnung für eine sich wandelnde Gesellschaft sein. Gerade auch deshalb, weil sie ihre klassische Aufgabe, die Literaturversorgung, in zunehmenden Maße online erfüllt. Es ist tatsächlich so, dass wir heute nicht mehr für jedes Buch und jede Fachzeitschrift in die Bibliothek gehen - viel häufiger finden wir unsere Informationen und unser Wissen im Netz. Aber es muss sich trotzdem lohnen, eine Bibliothek zu besuchen. Als Lernort, als Ort des Austausches und der Begegnung, und als Ort, wo ich nicht nur hingehe, wenn ich ein bestimmtes Buch suche, sondern der mir auch sonst eine Qualität des Aufenthaltes bietet, die ich anderswo nicht finde.

Genau aus diesem Grund ist die Rolle der Bibliotheken heute mindestens so wichtig wie vor 250 Jahren. Und um die Chancen zu nutzen, die sich aus der immer größeren und immer unüberblickbareren Menge an Wissen ergeben, brauchen wir Bibliotheken, die sich dieser neuen Aufgabe stellen und sie für sich und unsere Gesellschaft nutzen. So, wie es die WLB in den letzten Jahren getan hat. So wie Sie, lieber Herr Schaab, es von nun an erfolgreich fortsetzen werden.

Und Sie haben auch weitere große Aufgaben vor sich, beispielsweise den Bezug und die Inbetriebnahme des Erweiterungsbaus sowie die sich dann hoffentlich nahtlos anschließende Sanierung des Bestandsgebäudes. Lieber Herr Schaab, ich freue mich, dass wir mit Ihnen eine Persönlichkeit gefunden haben, die durch ihre langjährige Berufserfahrung die ideale Besetzung ist, um die vielfältigen Herausforderungen der Württembergischen Landesbibliothek anzugehen. Ich bin mir sicher, dass Sie die Potentiale der Landesbibliothek ausbauen, Ihre eigenen, persönlichen Akzente setzen und die mit der Digitalisierung gegebenen Möglichkeiten gestalterisch nutzen werden. Für Ihre Arbeit wünsche ich Ihnen und Ihrem ganzen Team weiterhin viel Kraft und Erfolg!

Vielen Dank!

Petra Olschowski

\section{Wissen teilen}

\section{Ein Dialog anlässlich der Amtseinführung zwischen Julia Hiller von}

\section{Gaertringen und Rupert Schaab}

Sehr geehrte Frau Staatssekretärin, sehr geehrte Damen und Herren, liebe Kolleginnen und Kollegen,

zunächst vielen Dank für die Einführung und die Unterstützung vielerseits in den letzten Wochen! Ich freue mich, dass Sie alle hierhergekommen sind, weil Sie das Interesse an der Arbeit der Württembergischen Landesbibliothek teilen. Wie die meisten wissen, gibt es zwei Landesbibliotheken in Baden-Württemberg, die Badische Landesbibliothek, für die Sie deren Direktorin, Dr. Julia Freifrau Hiller von Gaertringen hier neben mir begrüßen dürfen. Wir teilen uns die landesbibliothekarischen
Aufgaben hier im Land und wollen gemeinsam ein wenig über "Wissen teilen" sprechen, weil wir der Meinung sind, dass diese Devise die Ausrichtung beider Bibliotheken ganz gut zusammenfasst.

Julia Hiller von Gaertringen: Zu Beginn möchte ich Rupert Schaab als meinem neuen Bruder im baden-württembergischen Geschwister-Duo ganz herzlich zu seinem Amt gratulieren. Ich wünsche inm alles Gute für seine neuen Aufgaben, dass sein Schwung sich in Erfolg verwandle und dass er allzeit glücklich und zufrieden sein möge mit der wunderbaren Bibliothek, die ihm nun anvertraut ist. Wir haben vor Jahrzehnten als Kölner Referen- 